\title{
Delayed-interval BNT162b2 mRNA COVID-19 vaccination enhances humoral immunity and induces robust $\mathrm{T}$ cell responses
}

\author{
Victoria G. Hall ${ }^{1,4} \bowtie$, Victor H. Ferreira ${ }^{1,4}$, Heidi Wood², Matthew lerullo ${ }^{1}$, Beata Majchrzak-Kita1, \\ Kathy Manguiat ${ }^{2}$, Alyssia Robinson'², Vathany Kulasingam ${ }^{3}$, Atul Humar ${ }^{1,5}$ and Deepali Kumar ${ }^{1,5} \bowtie$
}

\begin{abstract}
Delayed dosing intervals are a strategy to immunize a greater proportion of the population. In an observational study, we compared humoral and cellular responses in health care workers receiving two doses of BNT162b2 (Pfizer-BioNTech) vaccine at standard (3- to 6-week) and delayed (8- to 16-week) intervals. In the delayed-interval group, anti-receptor-binding domain antibody titers were significantly enhanced compared to the standard-interval group. The $\mathbf{5 0} \%$ plaque reduction neutralization test (PRNT50) and PRNT90 titers against wild-type (ancestral) severe acute respiratory syndrome coronavirus 2 (SARS-CoV-2) and Alpha, Beta and Delta variants were higher in the delayed-interval group. Spike-specific polyfunctional $\mathrm{CD4}^{+}$and $\mathrm{CD8}^{+} \mathrm{T}$ cells expressing interferon- $\gamma$ and interleukin-2 were comparable between the two groups. Here, we show that the strategy of delaying second doses of mRNA vaccination may lead to enhanced humoral immune responses, including improved virus neutralization against wild-type and variant SARS-CoV-2 viruses. This finding has potentially important implications as vaccine implementation continues across a greater proportion of the global population.

Currently, a substantial portion of the world's population remains
\end{abstract} unvaccinated against coronavirus disease 2019 (COVID-19). Multiple vaccine types have been investigated and implemented worldwide to impede the spread of COVID-19, proving extremely successful in the prevention of infection and symptomatic disease $^{1}$. The BNT162b2 mRNA vaccine (Pfizer-BioNTech) has been authorized for administration with a 3-week interval between the two doses ${ }^{1}$. In Canada, a 4-month delay between vaccine doses was announced in March 2021 as part of a strategy to accelerate the proportion of the population that receives one dose and achieve higher overall protection in the context of limited vaccine supply. Shortages of COVID-19 vaccines continue in many jurisdictions, even as oversupply exists in others. Delayed-interval vaccine dosing is an unproven and controversial public health strategy whose effect on vaccine immunogenicity is not well studied, especially in relation to predominant circulating variants of concern (VOCs). VOCs have generally supplanted wild-type virus as the major strains causing infection in the population. A meta-analysis of two doses of the ChAdOx1 nCoV-19 vaccine has supported a delayed interval of 3 months between vaccine doses for improved vaccine efficacy and binding-antibody response ${ }^{2}$. More recently, it was found that delaying the administration of a second dose of BNT162b2 vaccine up to 6-14 weeks continues to provide strong cellular and humoral responses when compared to standard-interval vaccination ${ }^{3}$.

In our current study, a total of 93 health care workers (HCWs) were enrolled and received vaccination with two doses of the BNT162b2 vaccine from January to June of 2021 (Fig. 1a). Of these, 39 of $93(41.9 \%)$ received both doses of the vaccine at the standard interval and 54 of $93(58.1 \%)$ received both doses of the vaccine at the delayed interval (Fig. 1a). Median time to the second dose was 35 days in the standard-interval group (interquartile range (IQR), 21-35; full range, 21-38 days) and 88 days (IQR, 83-90; full range, 53-123 days) in the delayed-interval group. The median age of the cohort was 40.9 years (IQR, 34.5-52.5), and participants were predominantly female (74/93 (79.6\%)). Median age was similar between the two groups ( 40.3 years in the standard-interval group and 41.4 years in the delayed-interval group); female sex was also similarly distributed (31/39 (79.5\%) versus $43 / 54(79.6 \%)$ participants in the two groups, respectively). Blood work after the second dose of vaccine was taken at a median of 35 days (IQR, 29-40) and 32 days (IQR, 28-39) in the standard- and delayed-vaccination groups, respectively. Two participants had detectable anti-receptor-binding domain (anti-RBD) antibody prior to vaccination, although they did not have a history of documented COVID-19. No participant developed symptomatic COVID-19 infection after vaccination over the study period.

Therewere robusthumoralimmuneresponsesaftertwodosesofthe BNT162b2 vaccine in all participants. However, anti-RBD antibody titer was significantly greater (Fig. 1b) in the delayed-interval group compared to the standard-interval group (median: 10,816 $\mathrm{U} \mathrm{ml}^{-1}$ (IQR 6,642-16,695) versus 3,420 $\mathrm{U} \mathrm{ml}^{-1}$ (IQR, 1,792-7,765), respectively, $P<0.0001)$. This increase in titer had a moderately positive correlation to time interval (Fig. 1c) between the two doses (Spearman $r=0.501, P<0.0001$ ).

Neutralization titer was determined against wild-type and Alpha, Beta and Delta variants using PRNT assays. PRNT50 and PRNT90 represented the maximum dilution titers at which 50\% and $90 \%$ viral inhibition occurred, respectively. Prior to vaccination, all HCW s were negative for neutralization except for one HCW in the delayed-interval group who had a low positive neutralization titer of 1:20. After two doses of vaccine, all HCWs had positive neutralization with PRNT50 for wild-type, Alpha and Delta and 97.8\% for Beta. Geometric mean PRNT50 titers were significantly higher in the delayed-interval group against wild-type, Alpha, Beta and Delta

${ }^{1}$ Transplant Infectious Diseases and Ajmera Transplant Centre, University Health Network, Toronto, Canada. ${ }^{2}$ National Microbiology Laboratory \& Public Health Agency of Canada, Winnipeg, Canada. ${ }^{3}$ Department of Biochemistry, University Health Network, Toronto, Canada. ${ }^{4}$ These authors contributed equally: Victoria G. Hall, Victor H. Ferreira. ${ }^{5}$ These authors jointly supervised this work: Atul Humar, Deepali Kumar. ${ }^{凶}$ e-mail: Victoria.hall@uhn.ca; Deepali.kumar@uhn.ca 
a
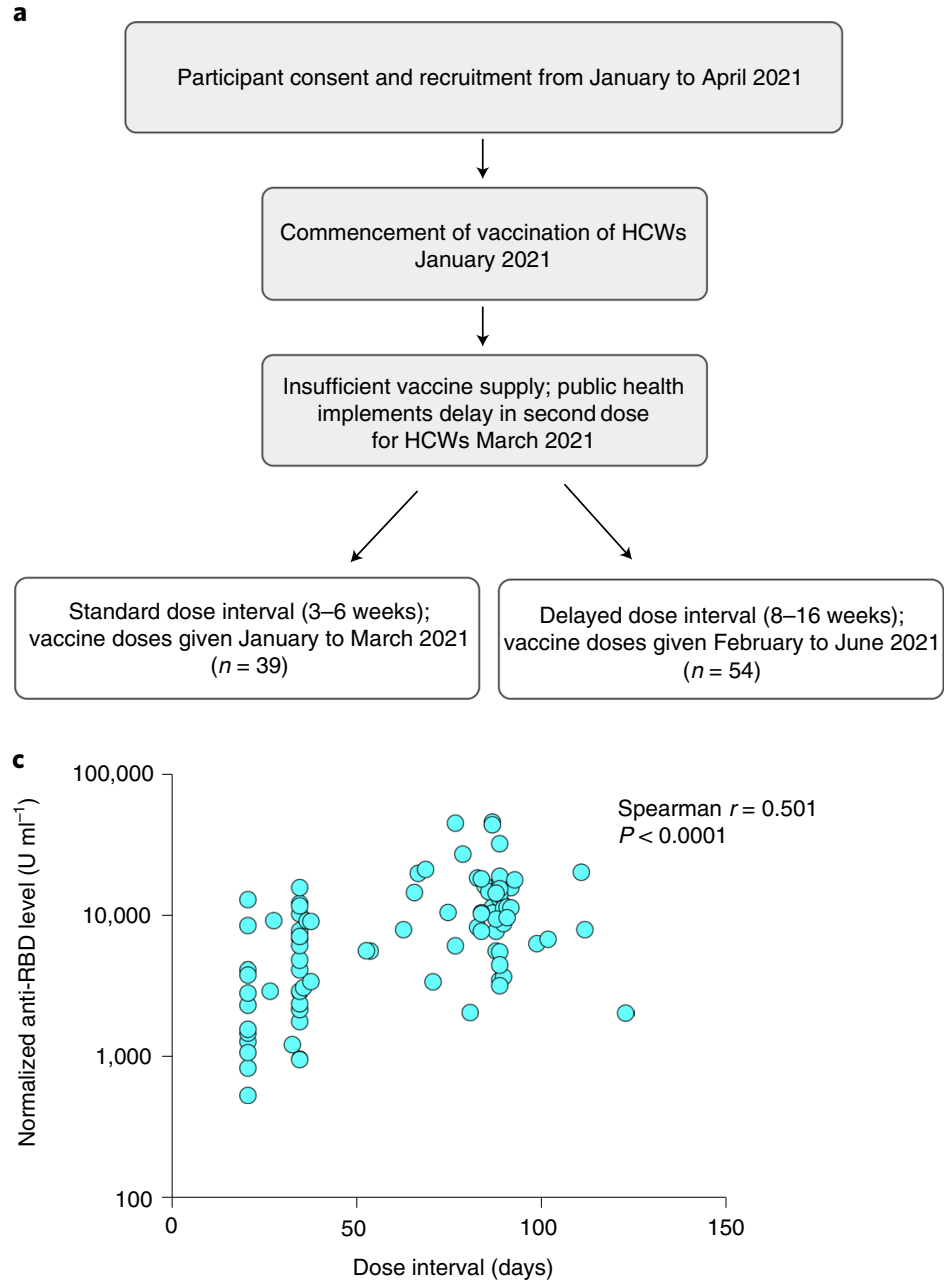

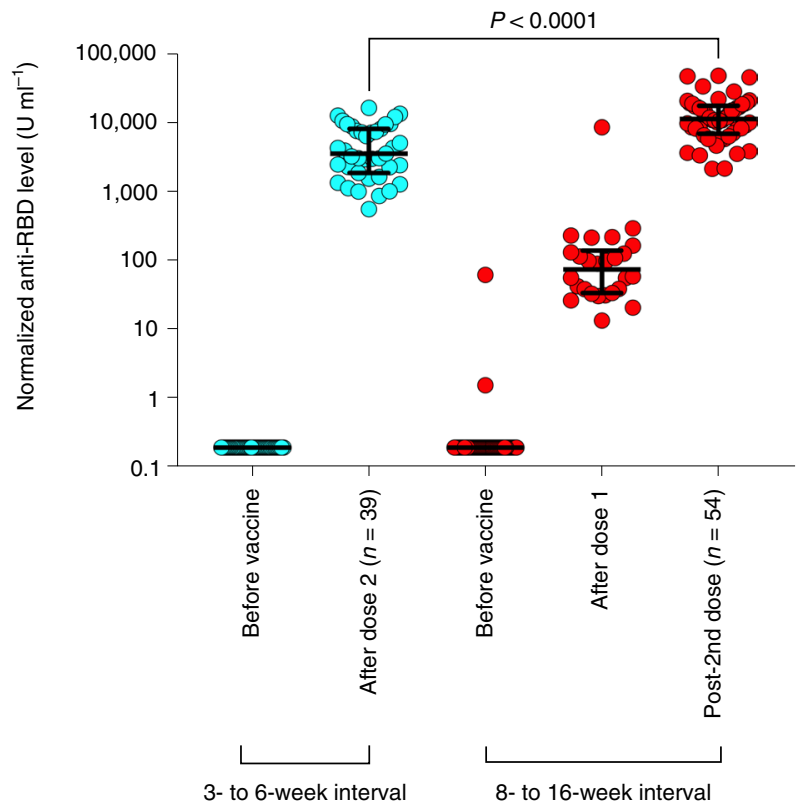

Fig. 1 | Study flow chart and anti-RBD titers between a standard (3-6 weeks) and delayed (8-16 weeks) second-dose interval in HCWs receiving two doses of BNT162b2 vaccine. a, Experimental design detailing time intervals of participant recruitment and dose intervals over the study period. b, Anti-RBD titer in standard $(n=39)$ and delayed $(n=54)$ groups $\left(\mathrm{U} \mathrm{ml}^{-1}\right)$. Each dot represents an individual participant. Horizontal lines represent median, and vertical lines indicate the IQR. Between-group comparisons were performed using a two-sided Mann-Whitney $U$ test. c, Correlation of anti-RBD titer with dose interval (in days) between doses 1 and 2 of the BNT162b2 vaccine. Spearman's correlation test was performed; Spearman $r$ and corresponding two-tailed $P$ values are shown in the top right corner.

variants of SARS-CoV-2 (Fig. 2a-e). PRNT90 titers were generally lower than PRNT50 titers (Fig. 3a-e), although the majority of HCWs had positive neutralization by both measures. For PRNT90, 98.9\% of HCWs had positive neutralization against wild-type, $94.6 \%$ for Alpha, 76.3\% for Beta and 91.4\% for Delta. Neutralization titers were significantly reduced against the Beta variant for both groups. Reverse cumulative distribution curves for each variant are shown and represent the proportion of participants reaching a threshold titer for PRNT50 and PRNT90 (Fig. 2b-e and Fig. 3b-e). These visually demonstrate improved responses in the delayed-interval group. Within the delayed-interval group, a comparison of post-first dose and post-second dose PRNT50 and PRNT90 is shown in Extended Data Fig. 1.

Cell-mediated immune responses were determined following stimulation with overlapping spike protein peptides. Spike-specific polyfunctional $\mathrm{CD}^{+} \mathrm{T}$ cells, defined by double positivity for interferon- $\gamma$ (IFN- $\gamma$ ) and interleukin-2 (IL-2) (Fig. 4a), were not significantly different in the delayed-interval group compared with the standard-interval group (median polyfunctional $\mathrm{CD}^{+} \mathrm{T}$ cell frequency: 208 (IQR, 128-407) versus 429 (IQR, 191-792) cells per $10^{6} \mathrm{CD}^{+} \mathrm{T}$ cells, respectively, Holm-Š́dák adjusted $\left.P=0.17\right)$.
The proportion of patients above the predefined cutoff of $0.01 \%$ (or 100 cells per million) was similar between the two groups $(97.4 \%$ standard interval versus $84.6 \%$ delayed interval, $P=0.156$ ), suggesting the majority of participants mounted spike-specific $\mathrm{CD} 4^{+} \mathrm{T}$ cell responses, regardless of vaccine interval. Polyfunctional $\mathrm{CD}^{+} \mathrm{T}$ cell responses tended to be lower than $\mathrm{CD}^{+}$responses in both cohorts (Fig. 4b), and overall, only 6 of 51 participants (11.8\%) elicited a vaccine-specific polyfunctional $\mathrm{CD}^{+} \mathrm{T}$ cell response if a threshold value of $\geq 0.01 \%$ was applied. When compared to PRNT50 and PRNT90 titers, polyfunctional CD4 ${ }^{+} \mathrm{T}$ cell responses were similar regardless of neutralizing antibody titer (Fig. 4c,d). Monofunctional $\mathrm{CD}^{+} \mathrm{T}$ cells expressing IL-2 in response to spike were lower in the delayed-vaccine group (Fig. 4a) (Holm-Š́lák adjusted $P=0.0052$ ). Monofunctional $\mathrm{CD}^{+} \mathrm{T}$ cells were readily detected in both groups (Fig. 4c) at similar levels.

Adverse events (AEs) for dose 2 were comparable between the standard- and delayed-interval groups (Fig. 5a,b). Specifically, systemic inflammatory events such as fever and chills occurred with comparable frequency in both groups. However, myalgia was reported more commonly in the delayed-interval group (32.0\% versus $15.8 \%$, respectively). Among those who provided 
a
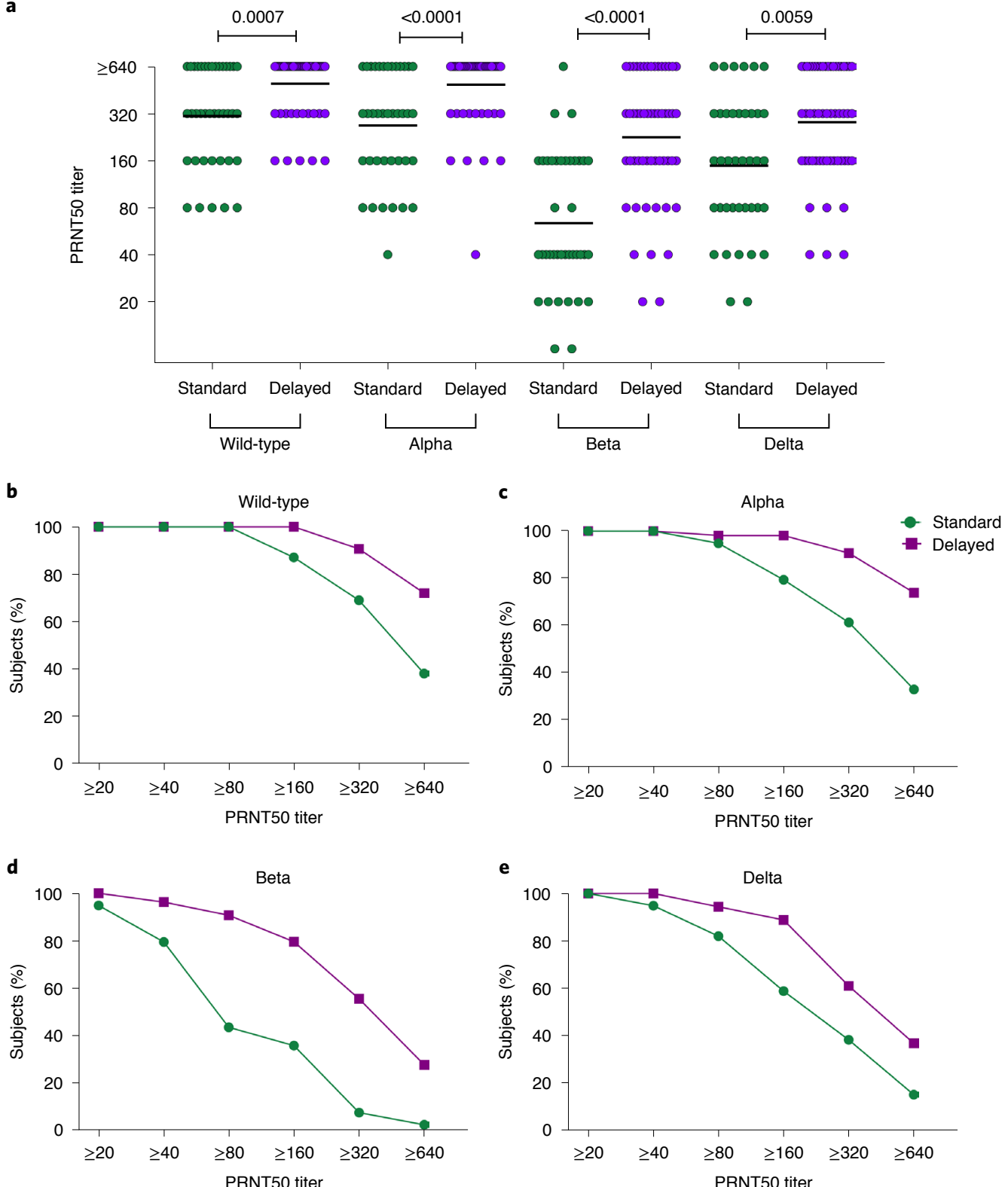

Fig. 2 | PRNT50 titers in participants receiving standard versus delayed vaccination. a, Comparative analysis of PRNT50 titers in standard ( $n=39)$ and delayed $(n=54)$ groups for wild-type and predominant circulating VOCs of SARS-CoV-2 (Alpha, Beta and Delta). Horizontal lines represent the geometric mean. Between-group comparisons were performed using a Mann-Whitney $U$ test; two-sided $P$ values are reported above each comparison. Participants with undetectable neutralizing antibodies were arbitrarily set to 10 (half of the lowest positive titer). b-e, PRNT50 reverse cumulative distribution curves, commencing at titers $\geq 20$, for the standard versus delayed group against wild-type (b), Alpha (c), Beta (d) and Delta (e) variants of SARS-CoV-2. Reverse cumulative distribution curves denote the percentage of participants that reach each titer threshold.

AE data $(n=77)$, the severity of AEs correlated with the degree of anti-RBD response. In those participants who experienced grade 2 events $(14 / 77(18.2 \%))$ with the second dose of vaccine (Fig. 5c), median anti-RBD antibody titer after the second dose was 14,617 $\mathrm{U} \mathrm{ml}^{-1}$ (IQR, 7,692-21,702) compared with 7,679 U/ml (IQR, $3,211-11,363)$ in those who experienced only grade 1 or no AEs $(P=0.0025)$.

Delayed dosing of mRNA COVID-19 vaccines has been a controversial issue, based primarily on pragmatic reasoning aimed at mitigation of vaccine shortages rather than scientific justification. The main findings of this comparative assessment of humoral and cellular immune response in HCWs receiving two doses of the BNT162b2 vaccine were as follows: (1) all HCWs, regardless of regular or delayed dosing intervals, produced robust immune responses after two doses of the vaccine; (2) anti-RBD titers were significantly higher in the delayed-interval group; (3) neutralizing antibody titers based on PRNT50 and PRNT90 were significantly higher in the delayed-interval group (this was observed for wild-type as well as Alpha, Beta and Delta SARS-CoV-2 variants); and (4) cellular immune responses represented by spike-specific monofunctional and polyfunctional $\mathrm{CD}^{+} \mathrm{T}$ cells, and $\mathrm{CD} 8^{+} \mathrm{T}$ cells were detectable in both groups and generally not significantly different, with the exception of monofunctional IL-2-producing $\mathrm{CD}^{+}$ $\mathrm{T}$ cells. The data presented provide scientific rationale to support delayed-dosing policies for mRNA vaccines, especially because there are limited peer-reviewed published data reporting on this. 
a
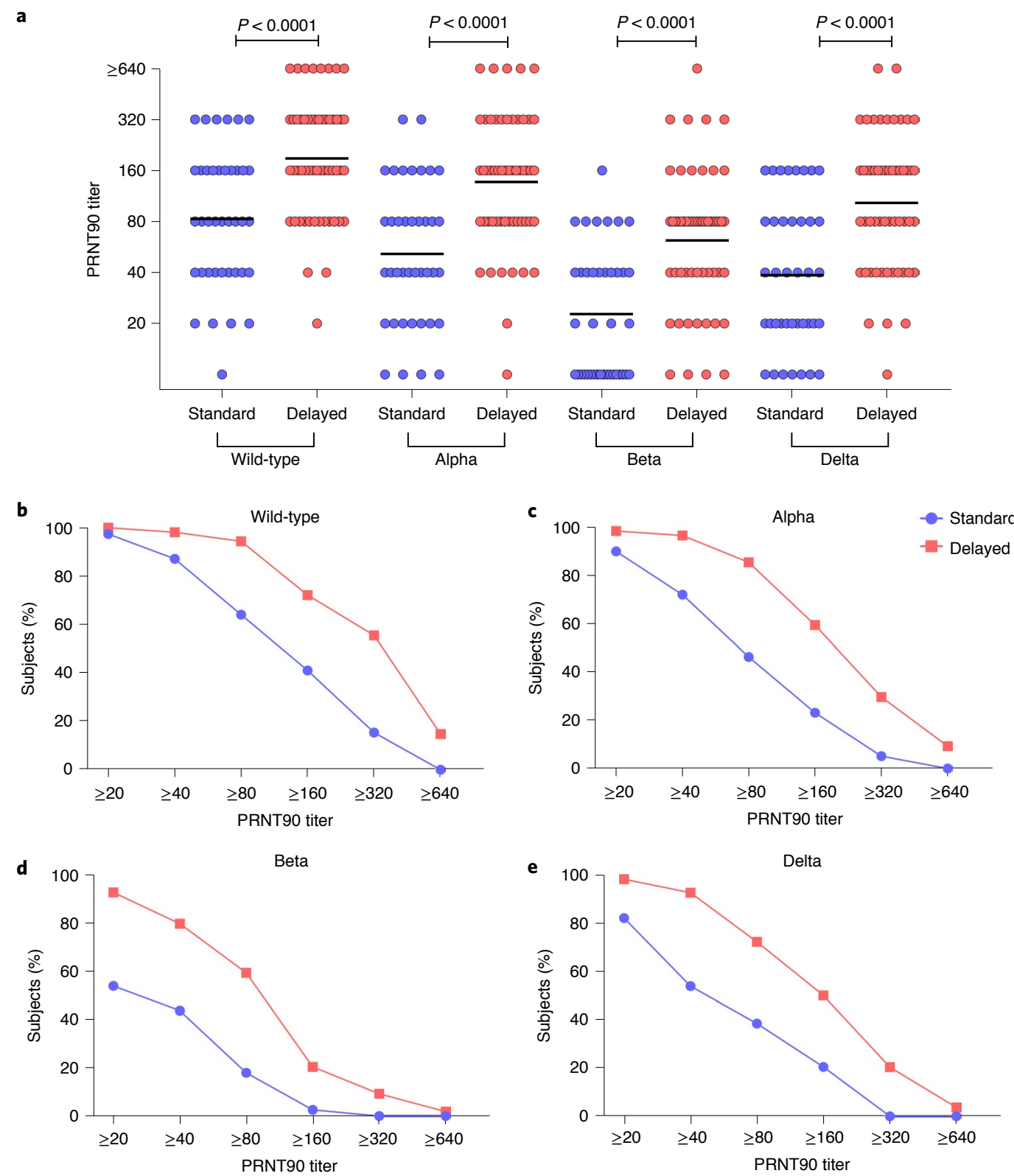

Fig. 3 | PRNT90 titers in participants receiving standard versus delayed vaccination. a, Comparative analysis of PRNT90 titers in the standard ( $n=39$ ) and delayed $(n=54)$ groups for wild-type and predominant circulating VOCs of SARS-CoV-2 (Alpha, Beta and Delta). Horizontal lines represent the geometric mean. Between-group comparisons were performed using the Mann-Whitney $U$ test; two-sided $P$ values are reported above each comparison. Participants with undetectable neutralizing antibodies were arbitrarily set to 10 (half of the lowest positive titer). b-e, PRNT90 reverse cumulative distribution curves, commencing at titers $\geq 20$, for the standard versus delayed group against wild-type (b), Alpha (c), Beta (d), and Delta (e) variants of SARS-CoV-2. Reverse cumulative distribution curves denote the percentage of participants that reach each titer threshold.

Concern has been raised that delaying the interval of the two doses of vaccine may encourage the development of new SARS-CoV-2 variants in the interim while individuals await the second dose $e^{4}$. This concern may be mitigated, however, by improved neutralization titers against circulating variants achieved in the delayed-vaccination group, possibly conferring a more durable response and longer-term protection, as well as potentially increasing the likelihood of sufficient antibody levels to confer efficacy against heterologous and partially vaccine-resistant variants. The greater humoral response achieved by delaying $\mathrm{mRNA}$ vaccination is consistent with studies of delaying the second dose of vector-based (ChAdOx1) vaccine ${ }^{2}$ and BNT162b2 (ref. ${ }^{3}$ ). Our study additionally provides PRNT90 data in addition to PRNT50 data. The former data may have better discriminatory ability in healthy cohorts. Also, we used flow cytometry (versus an enzyme-linked immunosorbent spot assay), which may allow for more detailed discrimination of cell populations and account for differences in our findings related to IL-2 monofunctional $\mathrm{CD} 4^{+} \mathrm{T}$ cells.

The mechanism resulting in enhanced antibody responses in the delayed vaccine group are unknown. It has been described previously $y^{5}$ that memory B cells against SARS-CoV-2 spike increase between 1 month and 8 months after infection, whereas memory $\mathrm{CD}^{+} \mathrm{T}$ cells decline. Similarly, it has been observed that by 6 months after infection, the number of RBD-specific memory B cells remains largely unchanged but displays greater resistance to RBD mutations and has increased potency $y^{6}$. This effect is likely a result of 

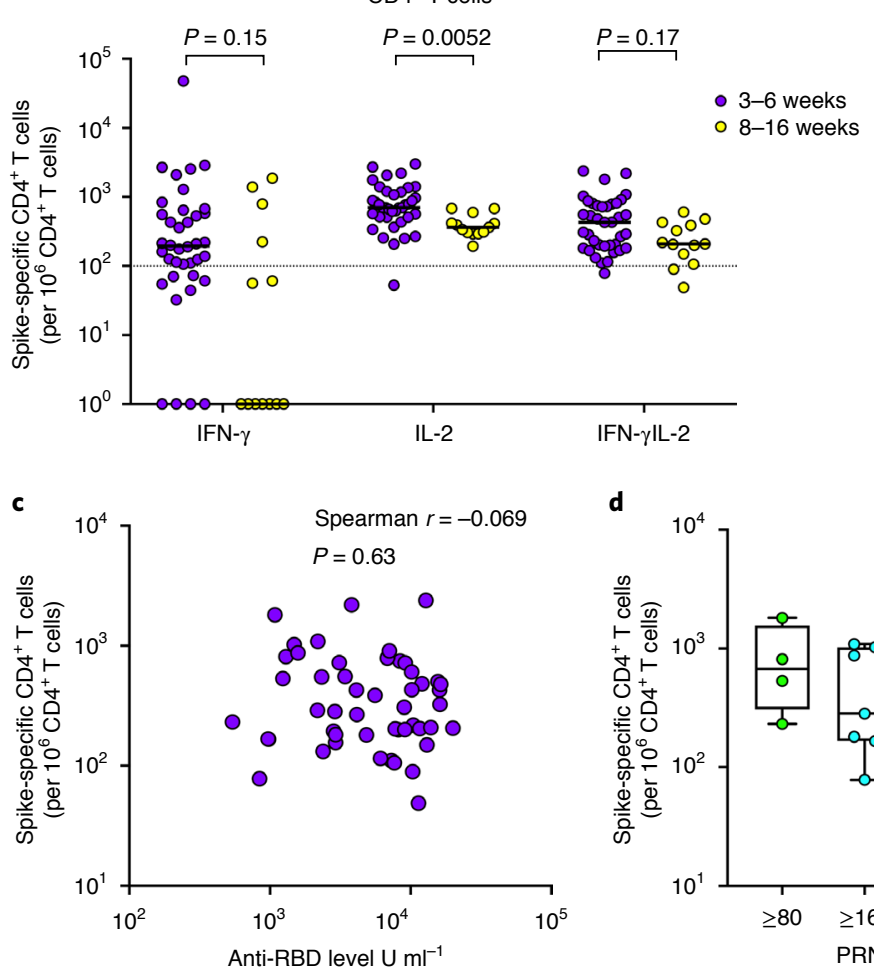

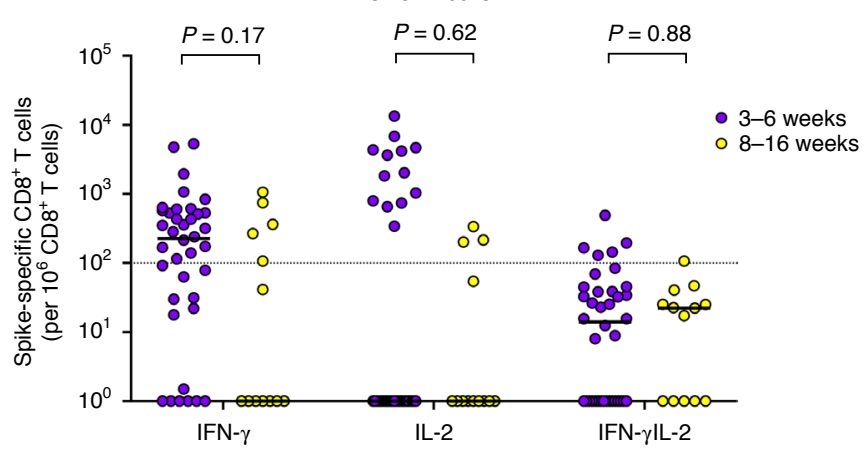

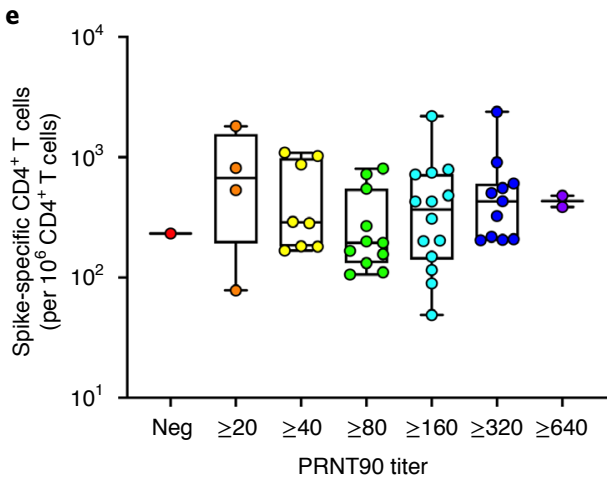

Fig. 4 | Spike-specific CD4 ${ }^{+}$and CD8 ${ }^{+}$T cell responses, and correlation of cellular with humoral responses. a,b, Proportions of IFN- $\gamma^{+}$monofunctional, $\mathrm{IL}-2^{+}$monofunctional and IFN- $\gamma^{+} \mathrm{IL}-2^{+}$polyfunctional $\mathrm{CD} 4^{+} \mathrm{T}$ cells $(\mathbf{a})$ and $\mathrm{CD} 8^{+} \mathrm{T}$ cells $(\mathbf{b})$ in $\mathrm{HCWs}$ with standard or delayed vaccination. Horizontal bars denote the median for each group. A two-sided Mann-Whitney $U$ test with Holm-Šídák correction for multiple comparisons was used. Adjusted $P$ values are shown above each respective comparison. Comparisons were performed between $n=38 \mathrm{HCWs}$ with standard vaccination and $n=13 \mathrm{HCWs}$ with delayed vaccination. c, Correlation of IFN- $\gamma^{+} \mathrm{IL}-2^{+}$polyfunctional $\mathrm{CD} 4^{+} \mathrm{T}$ cells versus anti-spike RBD levels $\left(\mathrm{U} \mathrm{ml}^{-1}\right)$. Spearman's correlation test was performed; Spearman $r$ and corresponding two-tailed $P$ values are shown in the top right corner. $\mathbf{d}, \mathbf{e}$, Box-whisker plots showing frequencies of IFN- $\gamma^{+}$IL-2 ${ }^{+}$ polyfunctional CD4 ${ }^{+}$T cells in $n=51 \mathrm{HCWs}$ according to corresponding PRNT50 (d) and PRNT90 (e) titers. Comparison across PRNT titers done using Kruskal-Wallis test, $P=$ NS (not significant). Plots show median (horizontal line), with the upper and lower bounds of the vertical lines indicating the maximum and minimum values, respectively. The upper and lower bounds of boxes represent the upper and lower limits of the IQR, respectively. In all plots, each participant is represented by a dot. Neg, negative PRNT90 result.

germinal center reactions in peripheral lymphoid tissues, where follicular helper $\mathrm{T}$ cells help guide processes like somatic hypermutation and affinity maturation that evolve the humoral response over time. Both mRNA vaccines have demonstrated potent SARS-CoV2-specific germinal center responses, even with just a single dose of vaccination ${ }^{7}$. As such, we propose that the memory B cell repertoire at the time of delayed vaccination may simply be of a greater caliber, leading to a higher quantity and/or quality of antibody response. Analogous maturation processes are not known to occur for T cells, which are thought to contract at a faster rate than $\mathrm{B}$ cells ${ }^{8}$. Although we did not measure memory B cells or follicular helper T cells in our study, we did identify lower frequencies of IL-2-monofunctional $\mathrm{CD}^{+}{ }^{+} \mathrm{T}$ cells in the delayed-immunization protocol. However, no statistical difference was measured between groups with respect to the polyfunctional $\mathrm{CD}^{+}$and $\mathrm{CD}^{+} \mathrm{T}$ cells, suggesting that delayed second-dose immunization is still capable of inducing robust vaccine-specific $\mathrm{T}$ cell responses. Importantly, no cellular correlate of immunity has been identified.

Our study has some limitations. Our cohort was younger and female predominant, and both factors can positively influence the immune response ${ }^{9}$. However, given similar age and sex distribution in the standard and delayed groups, this factor would not influence the group comparisons. Although we did not collect blood from the standard-interval group after the first dose of vaccine, there is no rationale to expect this to be different between the two groups (after the first dose). An analysis of circulating $\mathrm{T}$ follicular helper and spike-specific B cells was not performed owing to limited numbers of cells available for analysis. Our study was not powered to determine real-world vaccine effectiveness; however, in keeping with our results, recent Canadian epidemiologic data (in preprint at the time of writing) support improved vaccine effectiveness with the delayed second dose ${ }^{10}$. A strength was the duration of the study and follow-up after vaccination, with all participants observed for several months and at least 6 weeks after their second dose of vaccine.

In summary, our study demonstrates that a delayed-vaccination schedule does not appear to be detrimental to overall immunogenicity and actually results in improved neutralizing antibody responses to wild-type, Alpha, Beta and Delta variants. This is of public health importance in global jurisdictions, where significant portions of the population have yet to be vaccinated because of either vaccine unavailability or hesitancy. Our study also provides immunogenicity data to support recent epidemiologic observations ${ }^{10}$. Further studies of the significance of these findings, including the durability of the immune response over time, correlates of protective efficacy, and immunogenicity as it relates to heterologous variant viruses, would be helpful to define the optimal dosing interval and the necessity or timing of a booster dose of vaccine. Our cohort will be followed for 12 months after enrolment, with testing at 6 and 12 months after the second dose of vaccine, to provide longer-term assessment of the 
a

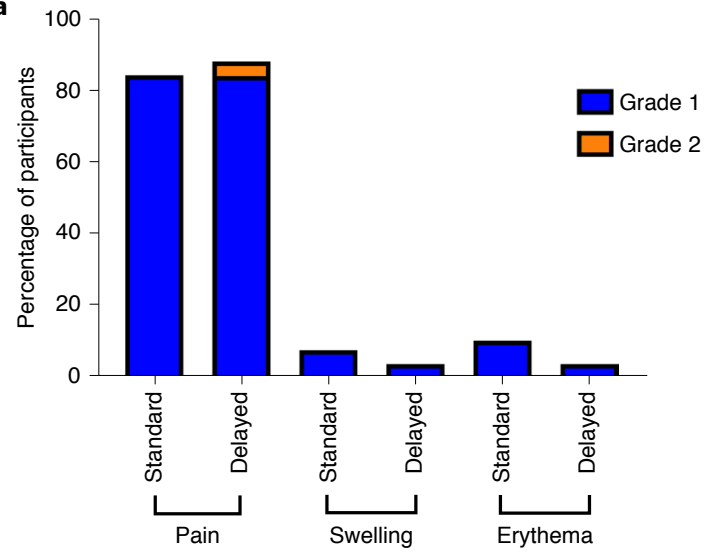

c

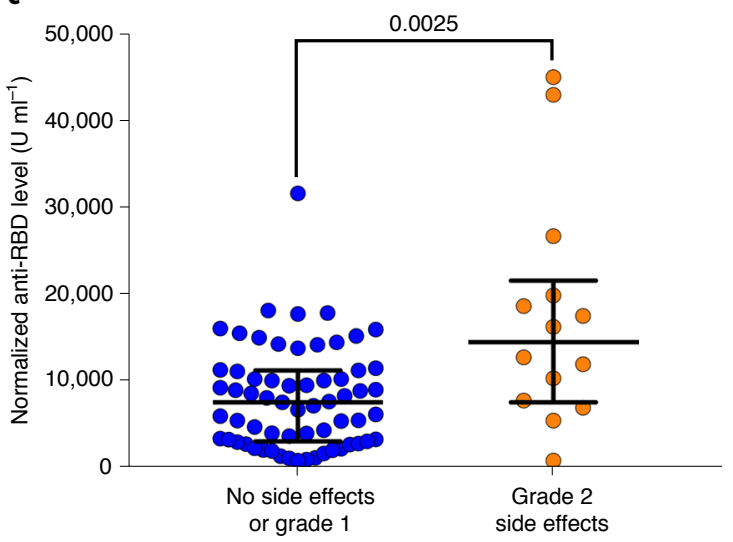

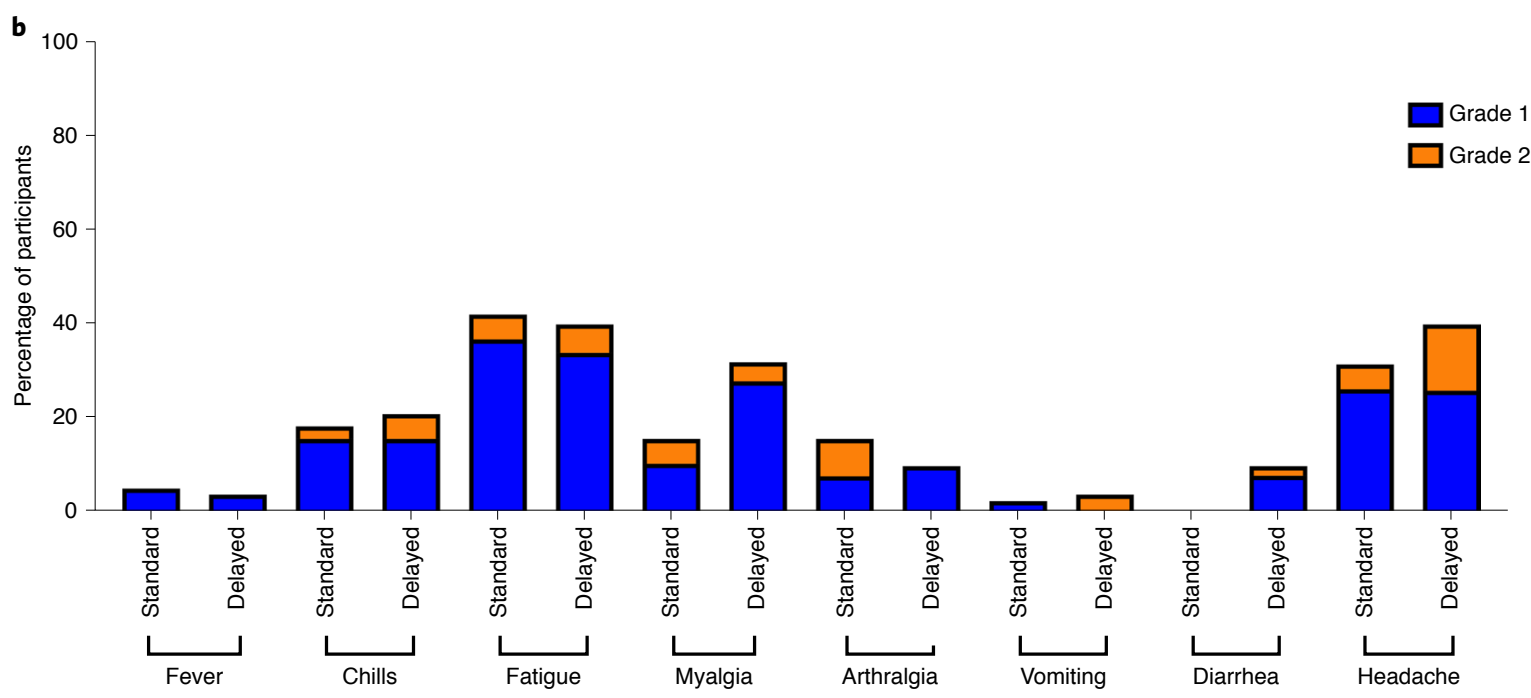

Fig. 5 | AEs from the second dose of BNT162b2 in the standard- (3-6 weeks) and delayed-interval (8-16 weeks) groups. a, Local AEs (grade 1 and 2) according to percentage of participants. b, Systemic AEs (grade 1 and 2) according to percentage of participants. c, Anti-RBD titer in participants with no or grade $1 \mathrm{AEs}(n=63)$ versus grade $2 \mathrm{AEs}(n=14)$. Horizontal lines represent median, and vertical lines indicate the IQR. Each participant is represented by a dot. Anti-RBD titer was analyzed using a Mann-Whitney $U$ test, and a two-sided $P$ value is shown for comparison.

effect of a standard versus delayed vaccination schedule on humoral and cell-mediated vaccine immunogenicity.

\section{Online content}

Any methods, additional references, Nature Research reporting summaries, source data, extended data, supplementary information, acknowledgements, peer review information; details of author contributions and competing interests; and statements of data and code availability are available at https://doi.org/10.1038/ s41590-021-01126-6.

Received: 10 November 2021; Accepted: 20 December 2021; Published online: 3 February 2022

\section{References}

1. Polack, F. P. et al. Safety and efficacy of the BNT162b2 mRNA COVID-19 vaccine. N. Engl. J. Med 383, 2603-2615 (2020).

2. Voysey, M. et al. Single-dose administration and the influence of the timing of the booster dose on immunogenicity and efficacy of ChAdOx1 nCoV-19 (AZD1222) vaccine: a pooled analysis of four randomised trials. Lancet 397, 881-891 (2021).
3. Payne, R. P. et al. Immunogenicity of standard and extended dosing intervals of BNT162b2 mRNA vaccine. Cell 184, 5699-5714 (2021).

4. Robertson, J. F. R., Sewell, H. F. \& Stewart, M. Delayed second dose of the BNT162b2 vaccine: innovation or misguided conjecture? Lancet 397, 879-880 (2021)

5. Dan, J. M. et al. Immunological memory to SARS-CoV-2 assessed for up to 8 months after infection. Science 371, eabf4063 (2021).

6. Gaebler, C. et al. Evolution of antibody immunity to SARS-CoV-2. Nature 591, 639-644 (2021)

7. Lederer, K. et al. SARS-CoV-2 mRNA vaccines foster potent antigen-specific germinal center responses associated with neutralizing antibody generation. Immunity 53, 1281-1295 (2020).

8. Macallan, D. C., Borghans, J. A. \& Asquith, B. Human T cell memory: a dynamic view. Vaccines (Basel) 5, 877 (2017).

9. Demonbreun, A. R. et al. COVID-19 mRNA vaccination generates greater immunoglobulin G levels in women compared to men. J. Infect. Dis. 224, 793-797 (2021).

10. Skowronski, D.M., et al. Two-dose SARS-CoV-2 vaccine effectiveness with mixed schedules and extended dosing intervals: test-negative design studies from British Columbia and Quebec, Canada. Preprint at medRxiv https://doi. org/10.1101/2021.10.26.21265397 (2021).

Publisher's note Springer Nature remains neutral with regard to jurisdictional claims in published maps and institutional affiliations.

(c) The Author(s), under exclusive licence to Springer Nature America, Inc. 2022 


\section{Methods}

Patient population and study design. This was a prospective observational cohort study of HCWs recruited from a tertiary, university-affiliated hospital network in Toronto, Canada, who were eligible to receive the two scheduled doses of the BNT162b2 (Pfizer/BioNTech) mRNA vaccine (Fig. 1a). Potential participants were recruited via mass emails to HCWs working at several hospital sites. Those who completed the two-dose vaccine series between 4 January 2021 and 13 June 132021 and consented were included and followed for at least 6 weeks after the second dose. Participants were excluded if they had known previous COVID-19 infection or had an immunosuppressive condition. Delayed dosing for HCWs came into effect in March 2021. This allowed for two study cohorts: those who received two doses at the standard interval (3-6 weeks) and those who received two doses at a delayed interval of $>8$ weeks. All participants provided blood samples prior to vaccination and 4-6 weeks after the second dose of vaccine. In the delayed-interval group, an extra blood sample was obtained 4 weeks after dose 1 . The local institutional research ethics board approved the study (University Health Network Research Ethics Board (CAPCR 20-6069)), and all participants provided signed informed consent.

Anti-RBD antibody and neutralization responses. Antibody against the spike RBD (anti-RBD) was measured using the commercial Roche Elecsys anti-SARS-CoV-2 S immunoassay. Briefly, the assay is a double-antigen sandwich electrochemiluminescence immunoassay that quantitatively detects antibodies to the RBD of the SARS-CoV-2 spike protein. Sera were tested as per the manufacturer's instructions in a certified biochemistry laboratory. The assay's lower limit of detection was $0.4 \mathrm{U} \mathrm{ml}^{-1}$, and a positive test result was defined as $\geq 0.8 \mathrm{U} \mathrm{ml}^{-1}$.

Neutralizing antibodies were assessed by PRNT50 and PRNT90 assays using ancestral (wild-type) SARS-CoV-2 (Canada/ON_ON-VIDO-01-2/2020, EPI_ISL_42517), titrated as previously reported ${ }^{11,12}$, as well as the SARS-CoV-2 variants B.1.1.7 (Alpha), B.1.351 (Beta) and B.1.167.2 (Delta). In a 96-well plate, antibodies were serially diluted and mixed with diluted SARS-CoV-2 (100 plaque-forming units (p.f.u.) per $100 \mu \mathrm{l}$ ), yielding final antibody dilutions of 1:20 to $1: 640$ and a final virus concentration of 50 p.f.u. per $100 \mu$ l. No neutralization, $50 \%$ neutralization and $90 \%$ neutralization controls were prepared by diluting SARS-CoV-2 at 50, 25, and 5 p.f.u. per $100 \mu$ l, respectively. We used $2 \%$ FBS and $1 \times$ penicillin supplemented DMEM as a no-virus control. After $1 \mathrm{~h}$ of incubation at $37^{\circ} \mathrm{C}$ and $5 \% \mathrm{CO}_{2}, 100 \mu \mathrm{l}$ of each antibody-virus mixture was added in duplicate to 12-well plates containing Vero E6 cells (ATCC; cell lines used were evaluated for Mycoplasma contamination via polymerase chain reaction and tested negative) at 95-100\% confluence, and $100 \mu \mathrm{l}$ of each control was added in triplicate to two sets of 12 -well plates containing Vero E6 cells. All plates were incubated at $37^{\circ} \mathrm{C}$ and $5 \% \mathrm{CO}_{2}$ for $1 \mathrm{~h}$ of adsorption, followed by adding liquid overlay containing equal volumes of $3 \%$ carboxymethylcellulose and $2 \times$ modified Eagle's medium (Temin's modification). After a 3-day incubation for the ancestral virus, Alpha and Beta and a 4-day incubation for Delta, the liquid overlay was removed and cells were fixed with $10 \%$ neutral-buffered formalin. The monolayer in each well was stained with $0.5 \%$ crystal violet $(\mathrm{w} / \mathrm{v})$, and the average number of plaques was calculated at each dilution and compared with the average number of plaques in the $50 \%$ and $90 \%$ neutralization controls. The reciprocal of the highest dilution resulting in $50 \%$ and $90 \%$ reduction in plaques compared with controls was defined as the PRNT50 and PRNT90 endpoint titers, respectively. PRNT50 titers and PRNT90 titers $\geq 20$ were considered positive for SARS-CoV-2 neutralizing antibodies, whereas titers $<20$ were considered negative.

Cell-mediated immunity assessment. In a subset of participants who provided additional blood, spike-specific $\mathrm{CD}_{4}^{+}$and $\mathrm{CD}^{+} \mathrm{T}$ cell responses were assessed as previously described ${ }^{13}$. Briefly, peripheral blood mononuclear cells (PBMCs) underwent Ficoll-based isolation and were cryopreserved in liquid nitrogen until use. A total of $10^{6} \mathrm{PBMCs}$ were rested for $2 \mathrm{~h}$ and subsequently incubated with 15-mer overlapping peptides encompassing the full SARS-CoV-2 spike protein (PepTivator, Miltenyi Biotec) at a final concentration of $5 \mu \mathrm{g} \mathrm{ml} l^{-1}$ per peptide, along with a CD28/CD49d costimulatory antibody cocktail (BD Biosciences) and a protein transport inhibitor to prevent cytokine release (Thermo Fisher Scientific). Cells were fixed, permeabilized and incubated with an antibody cocktail to detect intracellular markers (IFN- $\gamma$ and IL-2). PMA/ionomycin was used as a positive control, and cells with media alone were used as a negative control. Frequencies of $\mathrm{CD}^{+}$and $\mathrm{CD} 8^{+} \mathrm{T}$ cells that expressed both cytokines (polyfunctional cells) or only one cytokine (monofunctional) were measured. Flow cytometry was performed on an LSR II BGRV (BD Biosciences) at the SickKids-UHN Flow Cytometry Facility. The frequency of vaccine-antigen-specific $\mathrm{T}$ cells was determined by subtracting the frequencies of cytokine-positive $\mathrm{T}$ cells in unstimulated comparators from peptide-stimulated samples. A representative gating strategy is shown in Extended Data Fig. 2. A positive T cell response was defined at a frequency of $0.01 \%$ or greater (limit of quantitation). A minimum of 100,000 live $\mathrm{CD}^{+} \mathrm{T}$ cells were required for samples to be included in the flow analysis. Flow data were collected using BD FacsDiva version 6.1.3, and flow data were analyzed using FlowJo v10.7.1.
Antibodies used in this study were obtained from commercial vendors and included anti-human CD3-BV786 (clone SK7, 563799, BD Biosciences; dilution 1:80), anti-human CD4-Pacific Blue (clone RPA-T4, 558116BD, Biosciences; dilution 1:40) anti-human CD8-APC-Cy7 (clone RPA-T8, 557760, BD Biosciences: dilution 1:40), anti-human IFN- $\gamma$-FITC (clone B27, 554700, BD Biosciences; dilution 1:40), and anti-human IL-2-APC (clone MQ1-17H12, 500310, BioLegend; dilution 1:40). Validation of antibodies for specificity and application was directly performed by vendors, and corresponding data are available from the suppliers. Antibodies were titrated in-house to evaluate the best concentrations for discrimination of negative and positive cells using PBMCs collected from healthy human volunteers.

Safety and AEs. Safety assessments included monitoring through a participant-directed vaccine diary for local and systemic AEs for 7 days after each injection. AEs were categorized by the U.S. Food and Drug Administration toxicity grading scale for volunteers in vaccine trials as follows: grade 1 , no interference in daily activities; grade 2 , some interference in daily activities; grade 3 , participants unable to perform daily activities; and grade 4 , potentially life threatening ${ }^{14}$.

Statistical analysis. The primary analysis included post-second dose anti-RBD titers, neutralizing antibody and spike-specific polyfunctional and monofunctional $\mathrm{CD}^{+}$and $\mathrm{CD} 8^{+} \mathrm{T}$ cells between the standard- and delayed-interval groups. These were compared between groups using Mann-Whitney $U$ test, and a significant $P$ value was set at 0.05 . To adjust for multiple comparisons in the T cell analysis, the Holm-Šídák method was used to calculate adjusted $P$ values for monofunctional and polyfunctional $\mathrm{T}$ cells. Secondary analyses were as follows: (1) correlation between anti-RBD titer and time between doses using Spearman's test, (2) correlation between spike-specific polyfunctional $\mathrm{CD}^{+} \mathrm{T}$ cell response and anti-RBD level using Spearman's test, (3) a comparison polyfunctional $\mathrm{CD} 4^{+} \mathrm{T}$ cell response across PRNT50/PRNT90 titers using Kruskal-Wallis and (4) the median anti-RBD titer in those with grade 2 events compared to those no or grade 1 events using Mann-Whitney $U$ test. All measurements were taken from distinct samples, and all tests were two sided. Statistical analysis was performed using SPSS v29.0 and Prism GraphPad v9.1.1.

Reporting Summary. Further information on research design is available in the Nature Research Reporting Summary linked to this article.

\section{Data availability}

All numerical data found in the figures and extended data that support the findings of this study are fully available as source data files. Source data are provided with this paper.

\section{References}

11. Mendoza, E. J., Manguiat, K., Wood, H. \& Drebot, M. Two detailed plaque assay protocols for the quantification of infectious SARS-CoV-2. Curr. Protoc Microbiol 57, ecpmc105 (2020).

12. Valcourt, E. J. et al. Evaluating humoral immunity against SARS-CoV-2: validation of a plaque-reduction neutralization test and a multilaboratory comparison of conventional and surrogate neutralization assays. Microbiol. Spectr. 9, e0088621 (2021).

13. Hall, V. G. et al. Humoral and cellular immune response and safety of two-dose SARS-CoV-2 mRNA-1273 vaccine in solid organ transplant recipients. Am. J. Transpl. 21, 3980-3989 (2021).

14. Guidance for Industry: Toxicity Grading Scale for Healthy Adult and Adolescent Volunteers Enrolled in Preventive Vaccine Clinical Trials (U.S. Food and Drug Administration, 2007).

\section{Acknowledgements}

We thank N. Pinzon for expert study coordination and T. Ku for laboratory assistance. This work was supported by the Public Health Agency of Canada through the COVID-19 Immunity Task Force and Vaccine Surveillance Reference Group (D.K., A.H., V.G.H. and V.H.F.). V.G.H. has received research support funding from Avant Mutual, the Canadian Society of Transplantation and Ajmera Transplant Centre at University Health Network.

\section{Author contributions}

D.K., A.H., V.G.H. and V.H.F. designed the study; V.G.H., V.H.F., D.K. and A.H. analyzed data; V.G.H., V.H.F., M.I., B.M.-K., V.K., K.M., H.W. and A.R. performed the study; and D.K., A.H., V.G.H., V.H.F. and H.W. wrote the manuscript.

\section{Competing interests}

D.K. has received clinical trials grants from Roche and GSK and advisory fees from Roche, GSK, Sanofi, Merck and Exevir. A.H. has received a clinical trial grant from Roche and advisory fees from Merck. V.K. has received consultancy fees from Abbott 
Diagnostics. All competing interests are unrelated to the current study. The remaining authors declare no competing interests.

\section{Additional information}

Extended data is available for this paper at https://doi.org/10.1038/s41590-021-01126-6. Supplementary information The online version contains supplementary material available at https://doi.org/10.1038/s41590-021-01126-6.
Correspondence and requests for materials should be addressed to Victoria G. Hall or Deepali Kumar.

Peer review information Nature Immunology thanks Stephen Kent and the other, anonymous, reviewer(s) for their contribution to the peer review of this work. Zoltan Fehervari was the primary editor on this article and managed its editorial process and peer review in collaboration with the rest of the editorial team.

Reprints and permissions information is available at www.nature.com/reprints. 
A.

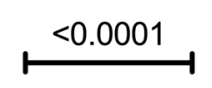

$\stackrel{<0.0001}{\longmapsto}$

$\stackrel{<0.0001}{\longmapsto}$

$\stackrel{<0.0001}{\longmapsto}$

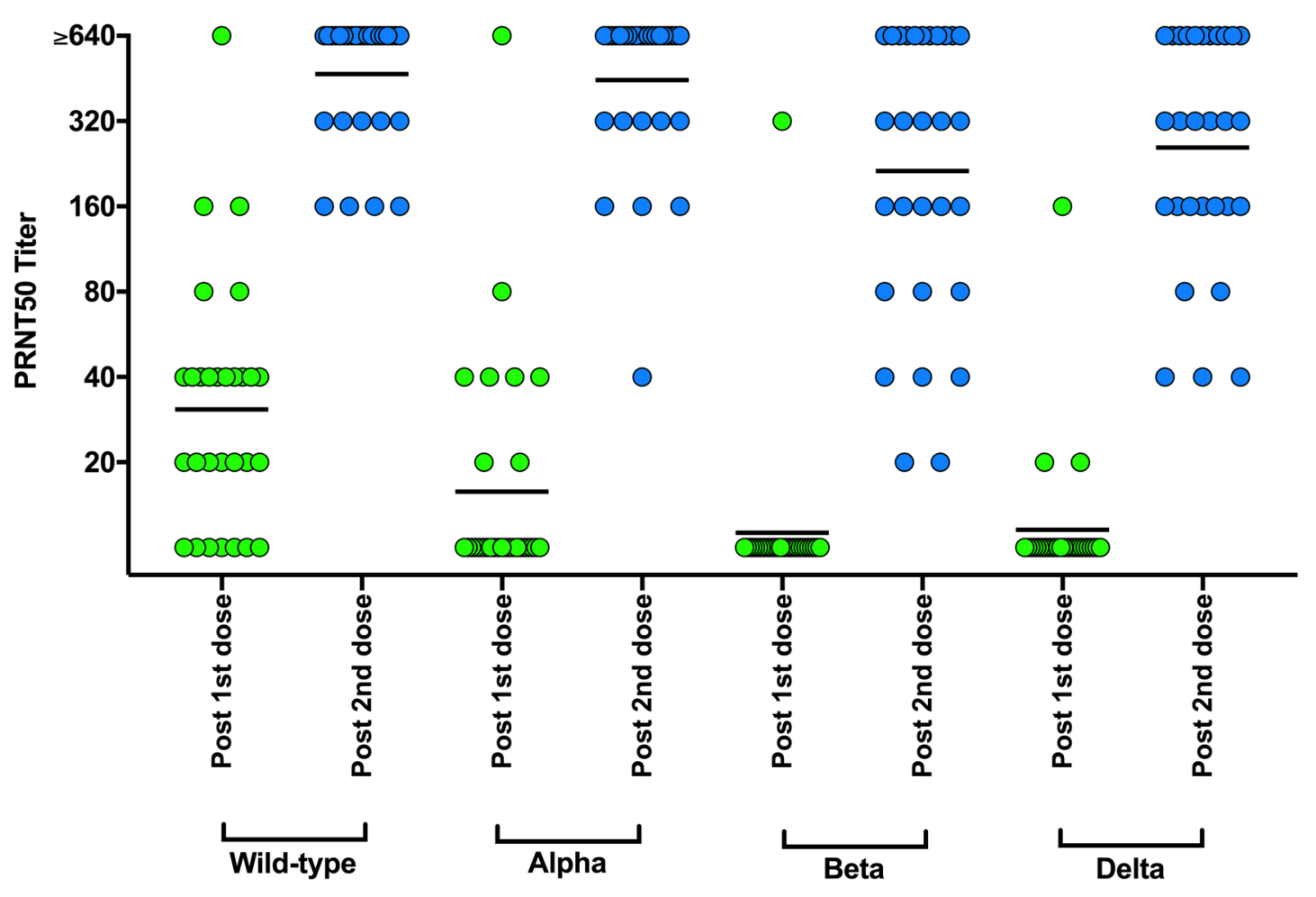

B.

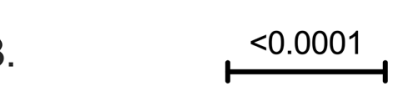

$$
\text { }
$$

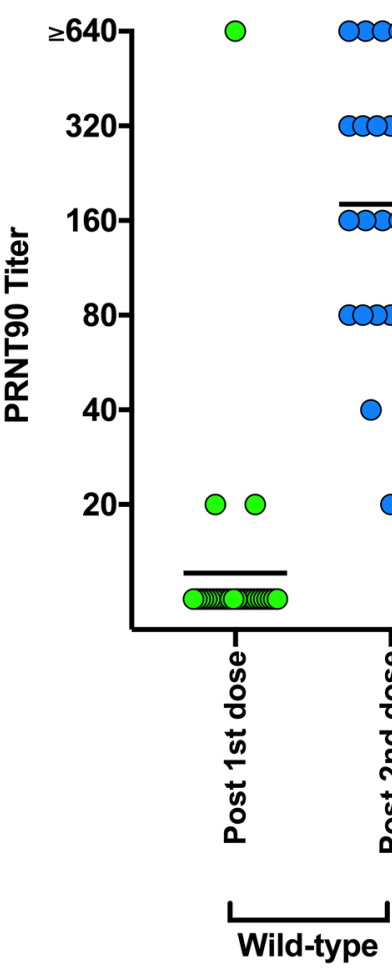

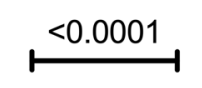

0000

000

ONoseco

00000

0000

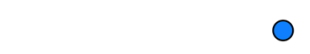

$\bigcirc$

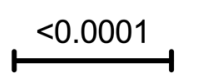

$\bigcirc$

$\bigcirc$

000

$\bigcirc$

Orsorseo $0 \overline{050050}$

000000

$\bigcirc \bigcirc 0$
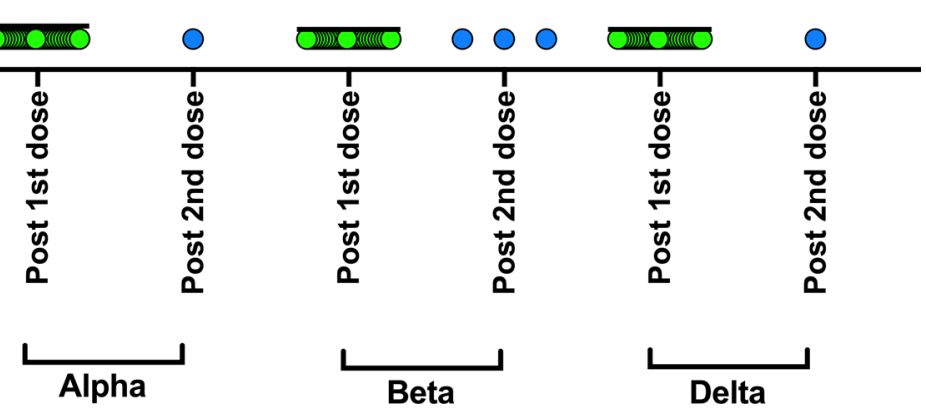
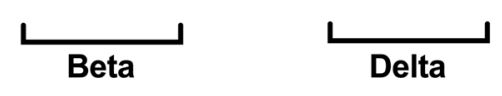

Extended Data Fig. 1 | See next page for caption. 
Extended Data Fig. 1 | PRNT titers after first and second dose in participants with delayed dosing. PRNT50 (A) and PRNT90 (B) titers in a subgroup of participants with delayed dosing who gave samples after both first-dose and second dose of vaccine. Comparisons against ancestral SARS-CoV-2, and variants of concern (alpha, beta and delta) are shown. Horizontal lines denote the median. Between group comparisons were performed using the Wilcoxon signed-rank test; two-sided p-values are shown above each corresponding comparison $(n=29)$. 
A
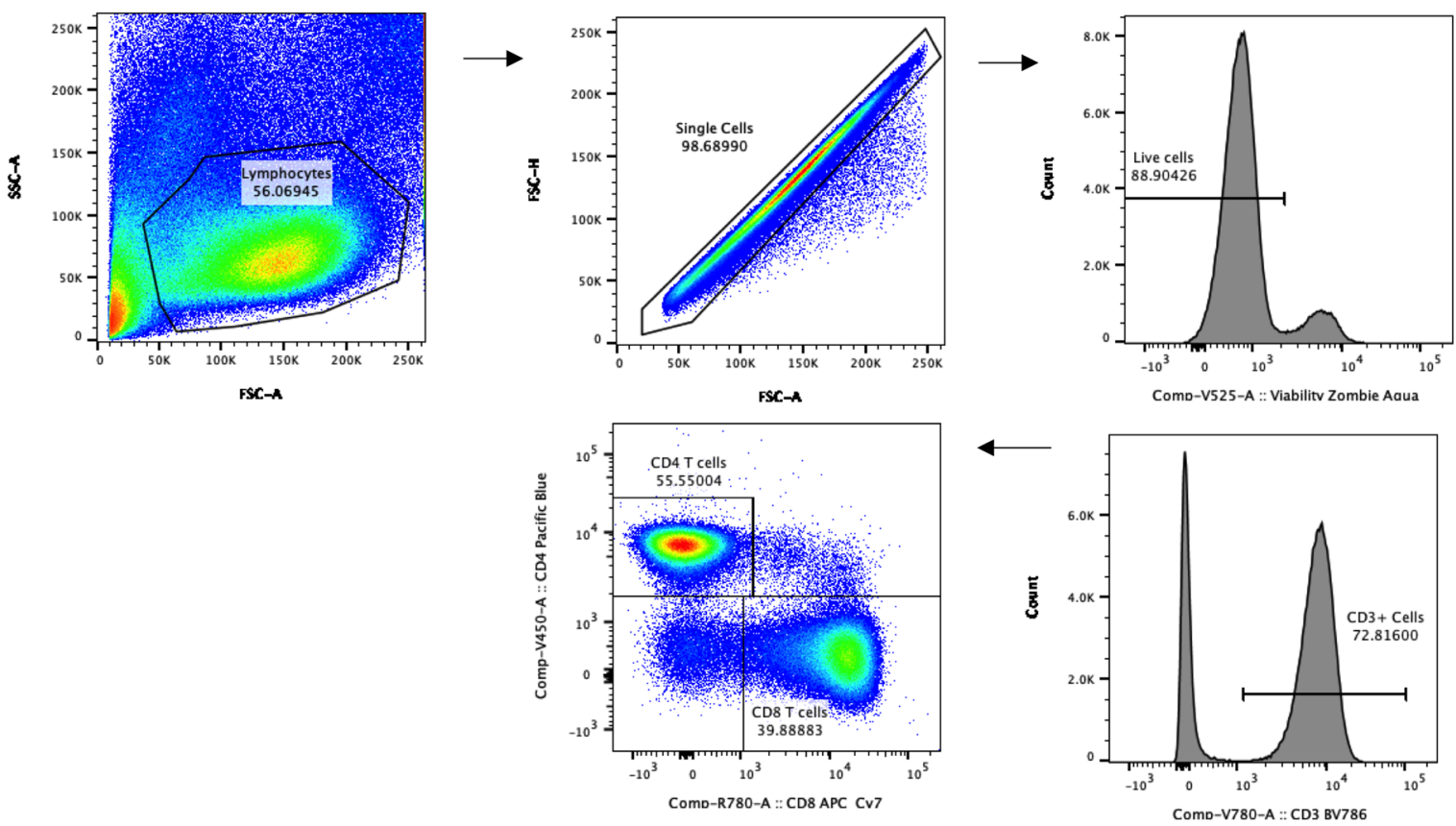

B

CD4+ T-cells

Pre-vaccination

Post-vaccination
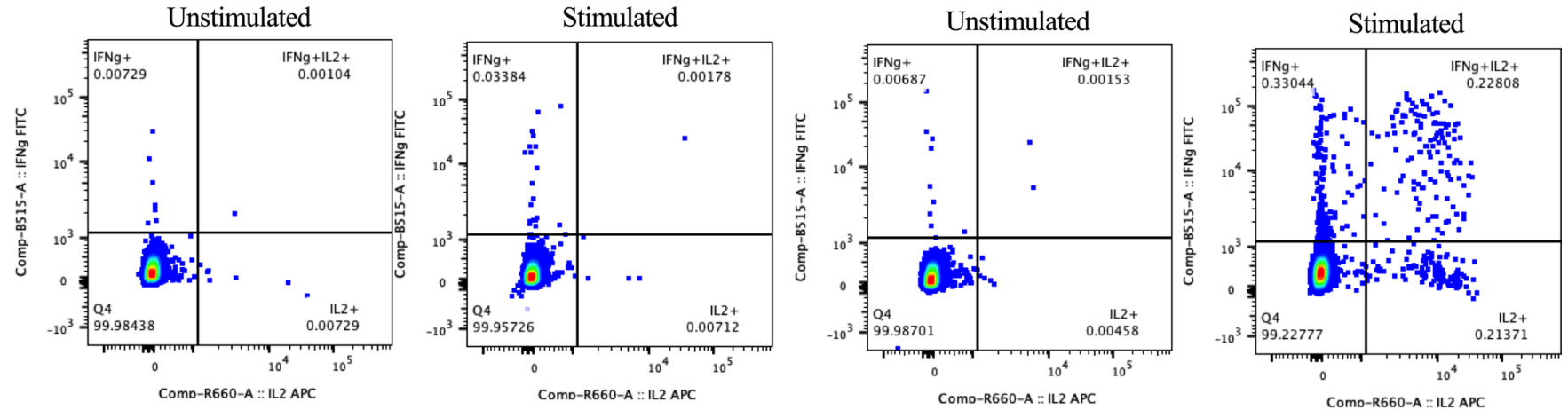

CD8+ T-cells

C

Pre-vaccination

Post-vaccination
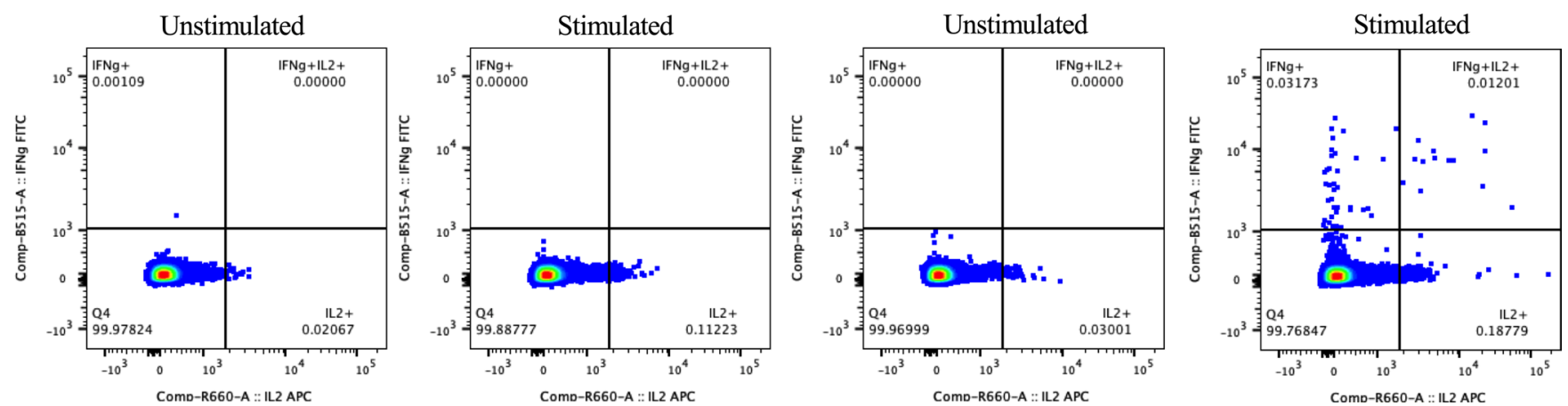

Extended Data Fig. 2 | See next page for caption. 
Extended Data Fig. 2 | Gating strategy and representative cytokine dot plots. (A) The sequential gating strategy for identifying CD4+ and CD $8^{+} T$ cells. Arrows indicate the sequence of hierarchical gates. Number in each plot refers to the frequency of the gated subset in the parental population. (B, C) Plots showing IFN- $\gamma$ (y-axis) vs. IL-2 (x-axis) production, gated on CD4+ $T$ cells (B) or CD8 ${ }^{+} T$ cells (C). Plot are taken from the same participant. Plots show $T$ cells stimulated with SARS-CoV-2 spike peptides, or media alone for the unstimulated control. Left two panels show response with 3-6-week interval, and right two panels show $T$ cell responses following administration of delayed (8-16-week interval). 


\section{Reporting Summary}

Nature Research wishes to improve the reproducibility of the work that we publish. This form provides structure for consistency and transparency in reporting. For further information on Nature Research policies, see our Editorial Policies and the Editorial Policy Checklist.

\section{Statistics}

For all statistical analyses, confirm that the following items are present in the figure legend, table legend, main text, or Methods section.

$\mathrm{n} / \mathrm{a}$ Confirmed

$\bigotimes$ The exact sample size $(n)$ for each experimental group/condition, given as a discrete number and unit of measurement

$\bigotimes$ A statement on whether measurements were taken from distinct samples or whether the same sample was measured repeatedly

$\varnothing$ The statistical test(s) used AND whether they are one- or two-sided

Only common tests should be described solely by name; describe more complex techniques in the Methods section.

Х $\square$ A description of all covariates tested

Х $\square$ A description of any assumptions or corrections, such as tests of normality and adjustment for multiple comparisons

$\triangle$ A full description of the statistical parameters including central tendency (e.g. means) or other basic estimates (e.g. regression coefficient)

AND variation (e.g. standard deviation) or associated estimates of uncertainty (e.g. confidence intervals)

For null hypothesis testing, the test statistic (e.g. $F, t, r$ ) with confidence intervals, effect sizes, degrees of freedom and $P$ value noted Give $P$ values as exact values whenever suitable.

Х $\square$ For Bayesian analysis, information on the choice of priors and Markov chain Monte Carlo settings

$\bigotimes \square$ For hierarchical and complex designs, identification of the appropriate level for tests and full reporting of outcomes

$\triangle \square$ Estimates of effect sizes (e.g. Cohen's d, Pearson's $r$ ), indicating how they were calculated

Our web collection on statistics for biologists contains articles on many of the points above.

\section{Software and code}

Policy information about availability of computer code

Data collection BD FacsDiva version 6.1.3

Data analysis FlowJo version 10.7.1.

SPSS version 29

Prism GraphPad version 9.1.1

For manuscripts utilizing custom algorithms or software that are central to the research but not yet described in published literature, software must be made available to editors and

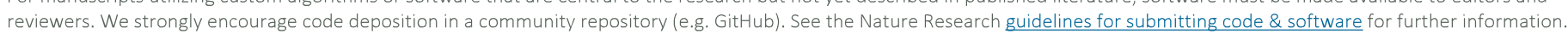

\section{Data}

Policy information about availability of data

All manuscripts must include a data availability statement. This statement should provide the following information, where applicable:

- Accession codes, unique identifiers, or web links for publicly available datasets

- A list of figures that have associated raw data

- A description of any restrictions on data availability

The data that support the findings of this study are available from the corresponding author upon reasonable request. 
Please select the one below that is the best fit for your research. If you are not sure, read the appropriate sections before making your selection.

\section{Life sciences study design}

All studies must disclose on these points even when the disclosure is negative.

Sample size

The healthcare worker was recruited as part of a larger study looking at COVID-19 vaccine immunogenicity in transplant patients. In the larger study, the sample size for healthy controls was 120 for comparison to transplant patients. However, because vaccine in Canada was delayed for healthcare workers, this control group could no longer be compared to the transplant group. For the analysis presented here, no formal sample size calculation was performed since the recommendations about the interval between first and second dose of COVID-19 vaccine for healthcare workers were rapidly changing in Canada due to vaccine shortages, and it was unclear how many healthcare workers would be vaccinated at the standard vs. delayed intervals (real world recruitment with convenience sampling).

Data exclusions Healthcare workers with prior documented COVID-19 infection were excluded.

Replication Binding Antibody - For antibody testing, this was done using commercially validated assays in a CLIA-certified biochemistry lab. Live virus neutralization - The neutralization assay was done at the National Microbiology Laboratory/Public Health Agency of Canada using a validated plaque reduction neutralization test. This is a central reference lab performing live-virus PRNT testing for Canada.

T cell assays - Steps were taken during the performance of the T-cell assays to ensure robustness including having an adequate number of live cells. All the assays used in this study including T-cell assays have been previously published by our group in peer-reviewed journals (most recently Hall et al., NEJM, Aug 11, 2021). No replicates were performed due to limited sample material.

Randomization Not applicable. This was a cohort study and therefore was not randomized. It was not possible to randomize due to uncertainties in vaccine distribution.

\section{Reporting for specific materials, systems and methods}

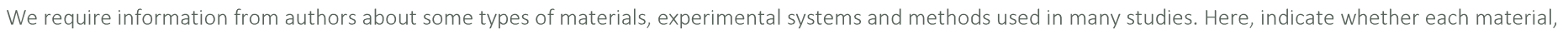

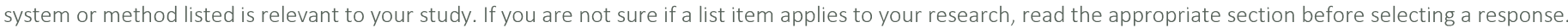

\begin{tabular}{l|l} 
Materials \& experimental systems \\
\hline$n / a$ & Involved in the study \\
\hline & $\bigotimes$ Antibodies \\
$\square$ & $\bigotimes$ Eukaryotic cell lines \\
$\square$ & $\square$ Palaeontology and archaeology \\
$\square$ & $\square$ Animals and other organisms \\
$\square$ & $\square$ Clinical data \\
\hline
\end{tabular}

\begin{tabular}{l|l} 
Methods \\
\hline n/a & Involved in the study \\
$\square$ & $\square$ ChIP-seq \\
$\square$ & $\bigotimes$ Flow cytometry \\
$\searrow$ & $\square$ MRI-based neuroimaging
\end{tabular}

\section{Antibodies}

Antibodies used

Flow cytometry:

anti-human CD3-BV786 (BD Biosciences) clone SK7, cat no: 563799, dilution 1:80

anti-human CD4-Pacific Blue (BD Biosciences) clone RPA-T4, cat no: 558116, dilution: 1:40

anti-human CD8-APC-Cy7 (BD Biosciences) clone RPA-T8, cat no: 557760, dilution: 1:40

anti-human IFN- $\gamma$-FITC (BD Biosciences) clone B27, cat no: 554700, dilution: 1:40

anti-human IL-2-APC (BioLegend) clone MQ1-17H12, cat no: 500310, dilution: 1:40

Validation

All antibodies were obtained from commercial vendors and corresponding Data Sheets are available and provided by the manufacturers describing validation of specificity. All antibodies were separately titrated in-house to evaluate best concentrations for discrimination of negative and positive cells. 
Policy information about cell lines

Cell line source(s)

Vero E6 (purchased through ATCC)

Authentication

Cell lines were authenticated by the provider. Additional information about the cell line can be found at the following link: https://www.atcc.org/products/crl-1586.

Mycoplasma contamination

Cell lines were tested for Mycoplasma (and were negative by PCR).

Commonly misidentified lines (See ICLAC register)

No commonly misidentified cell lines were used.

\section{Human research participants}

\section{Policy information about studies involving human research participants}

Population characteristics

We prospectively recruited immunecompetent healthcare workers at the University Health Network, Toronto, who intended to receive two doses of COVID-19 vaccine. The 93 participants who enrolled in the study were predominantly female (79.6\%) and median age was 40.9 years. Initially, vaccine was provided at the standard interval as stated in the product monograph; however, due to limited supply, second doses for healthcare workers were delayed for up to 16 weeks. Therefore, 39/93 participants received vaccine at standard intervals and 54/93 participants had a delay in their second dose.

Recruitment

Participants were recruited by sending a mass email to healthcare workers. This was a prospective observational study and all participants provided written informed consent. The study cohort represents the demographics of healthcare workers at our institution and there is no apparent self-selection bias.

Ethics oversight

University Health Network Research Ethics Board

Note that full information on the approval of the study protocol must also be provided in the manuscript.

\section{Flow Cytometry}

\section{Plots}

Confirm that:

\The axis labels state the marker and fluorochrome used (e.g. CD4-FITC).

ХThe axis scales are clearly visible. Include numbers along axes only for bottom left plot of group (a 'group' is an analysis of identical markers).

\All plots are contour plots with outliers or pseudocolor plots.

$\bigotimes$ A numerical value for number of cells or percentage (with statistics) is provided.

Methodology

Sample preparation

Instrument

Software

Cell population abundance

Gating strategy
PBMCs were isolated using conventional Ficoll-based separation protocols. After isolating the PBMCs from the peripheral blood, they were treated for $15 \mathrm{~min}$ in red cell lysis buffer according to the manufacturer's instruction (BD Pharmalyse). Cells were cryopreserved in 10\% DMSO + 90\% FBS (ThermoFisher Scientific). Cells were stored in a Mr. Frosty Freezing Container (ThermoFisher Scientific) for 24 hours at -80 oC prior to storage in the vapor phase of liquid nitrogen. Samples were assessed in batches. Cells were gently thawed in a $370 \mathrm{C}$ water bath and washed in complete RPMI ( $10 \%$ FBS, L-glu, pen/strep, HEPES) to remove residual DMSO. Cells were counted using a Vi-Cell instrument (Beckman Coulter) and seeded in 96-well plates at 1 million cells per well in complete RPMI. Cells were rested for 2 hours at $370 \mathrm{C}$ prior to undergoing stimulation with/without peptides. Peptides were added at $5 \mathrm{ug} / \mathrm{mL}$, as determined by prior experiments and co-stimulatory antibodies were added (BD FastImmune anti-CD28/CD49d). An hour after stimulation of cells, a protein transport inhibitor was added to prevent cytokine release (ThermoFisher Scientific). Cells were incubated overnight (16 hours) and subsequently stained for viability using the Zombie Aqua viability dye (BioLegend) at 1:1000 dilution. Cells were Fc blocked (BD Biosciences), and stained with a surface antibody cocktail consisting of human anti-CD3,-CD4 and -CD8 antibodies for $30 \mathrm{~min}$ on ice. Cells were then fixed in 4\% paraformaldehyde (Fixation Buffer, BioLegend, Cat no: 420801) at room temperature for $30 \mathrm{~min}$. Cells were incubated with an intracellular antibody cocktail targeting anti-human IFN- $\gamma$ and IL-2 prepared in Intracellular Staining Permeabilization Wash Buffer (BioLegend) for $20 \mathrm{~min}$ at room temp. After staining, cells were washed thoroughly and resuspended in wash buffer. Tubes were wrapped in foil to protect from light, and kept at $40 \mathrm{C}$ until acquisition of data. Data was acquired within 24 hours of staining.

Data was analyzed on an LSR II VBGR instrument (Becton Dickinson) at the SickKids-UHN Flow Cytometry Core Facility.

Acquisition was performed using BD FACSDiva. Analysis was performed using FlowJo, v10.7.1.

N/A. No sorting was performed.

Gating Strategy: SSC-A vs FSC-A (lymphocytes) -> FSC-H vs FSC-A (single cells) -> count vs viability-Zombie Aqua (live cells; 
gated on Zombie Aqua-negative cells) -> count vs CD3-BV786 (CD3+ T-cells; gated on CD3+ cells) -> CD4-pacific blue vs CD8APC-Cy7 (CD4+ T-cells are CD8-negative, and CD8+ T-cells were CD4-negative). Live CD4+ and CD8+ T-cells were then gated in terms of IFN- $\gamma$-FITC vs IL-2-APC to display cytokine production. To gate live/dead cells properly, control specimens were prepared where PBMCs were heat-killed by incubation at $650 \mathrm{C}$ for $10 \mathrm{~min}$ and then mixed in a 1:1 ratio with live (non-heat killed cells). These cells were then stained for flow analysis. To determine the proper placement of cytokine gates for determining cut-offs, a combination of negative controls (unstimulated PBMCs) and positive controls (PBMCs stimulated with PMA/ionomycin for 16 hours, a known potent inducer of IFNg and IL2 production in T-cells) were used.

$\bigotimes$ Tick this box to confirm that a figure exemplifying the gating strategy is provided in the Supplementary Information. 